\title{
Herpes Simplex Virus Encephalitis
}

\section{Lt Col A Padwell}

FRCS, MRCPath, RAMC

Senior Specialist in Pathology

Queen Elizabeth Military Hospital, Stadium Road, Woolwich, London SE184QH

SUMMARY: The clinico-pathological features, including autopsy findings, of two cases of delayed diagnosis of herpes simplex virus encephalitis presenting in young soldiers are reviewed, along with a discussion of the attendant diagnostic problems.

\section{Introduction}

Herpes simplex virus encephalitis is one of the more common sporadic viral encephalitides with an estimated incidence of one case per half million individuals per year $(1,2)$, but the true incidence may be higher. Untreated it is associated with a mortality rate exceeding $70 \%$ and a high morbidity amongst the survivors with only about $9 \%$ returning to normal neurological function. It is now accepted that acyclovir is the treatment of choice and with timely treatment the mortality can be reduced to $20 \%$ or less with a corresponding reduction in morbidity amongst the survivors $(1,3,4,5)$.

Two cases are reported involving previously fit soldiers who presented in BAOR with eventual fatal outcomes, and reinforces the need for prompt treatment if such an outcome is to be avoided.

\section{Case Reports}

\section{Case 1}

A previously fit and healthy 32 year old male presented to his Medical Reception Station (MRS) in Germany on 9 March 1988 complaining of sore throat and fever of a few days duration. A diagnosis of tonsillitis was made and he was admitted for observation. Later that day he became confused with a change in behaviour. The following day he was transferred to hospital where he suffered a grand mal fit.

On 14 March a computerised tomogram (CT) scan was arranged which showed a left temporal/parietal lesion suggestive of tumour. He was casevaced back to the United Kingdom on 15 March, by which time his condition had further deteriorated requiring intubation and ventilation.

On 16 March a repeat CT scan showed extensive swelling of the left temporal lobe thought to be inflammatory rather than neoplastic and herpes encephalitis was suggested. Brain biopsy and smear were performed. No tumour was identified but necrotic brain tissue was demonstrated. Immunocytochemistry for herpes simplex virus was positive.

He was commenced on acyclovir which was discontinued on 30 March. He made some initial recovery to the extent that he could feed himself and was able to talk, but was generally mentally retarded with severe behavioural problems.
In August he was transferred to Edinburgh for re- के habilitation nearer to his family, some 5 months after the $\vec{\circ}$ onset of his illness.

In October he was returned back to the hospital still $\vec{\omega}$ mentally retarded with behavioural problems.

On 6 July 1989 , he developed a pyrexia of $38^{\circ} \mathrm{C}$, a tachycardia of $140 / \mathrm{min}$ and became tachypnoeic and cyanosed. Chest X-ray revealed a right basal pneumonia $\vec{\omega}$ and blood culture a growth of pneumococcus. He was treated with amoxycillin but died on 8 February, about N 11 months after his initial illness.

\section{Case 2}

A 23 year old previously fit soldier on returning fromit exercise on 26 July 1991 presented to his medical cent in Germany complaining of headache and feeling unweft for about one week. His symptoms worsened and ke attended a local Krankenhaus that evening: he was givon. antipyretics and advised to return to his medical cente the following morning. That night he became confused and feverish and given paracetamol by this fiancée. At $\frac{4}{4} \overline{0}$

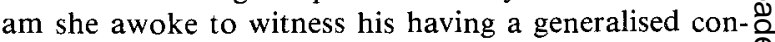
vulsion. She called an ambulance to take him to the local $\stackrel{\mathbb{Q}}{\varrho}$ Krankenhaus where he suffered a second generalised $\overrightarrow{\overrightarrow{0}}$ convulsion. A lumbar puncture revealed a leucocytosis. $\exists$ A CT scan was performed which was said to be normal.

A presumptive diagnosis of bacterial meningitis was. made and he was commenced on penicillin and refobacin. He persisted with a fever of $40^{\circ} \mathrm{C}$, developed personality changes and became confused.

He was transferred to hospital on 29 July: and on $\overline{8}$ admission was found to be stuporose with no verbal responses. In view of the lack of response to antibiotics, $\vec{F}$ herpes simplex encephalitis was considered a possible $\frac{3}{3}$ diagnosis and acyclovir was commenced.

The following day he suffered a respiratory arrest. $\mathrm{He} O$ was resuscitated but required ventilatory support. $\mathrm{He}$ remained in deep coma.

On 30 July examination of the cerebrospinal fluid 7 (CSF) revealed positive serology for herpes simplex virus with an IgM titre of $1 / 640$ and IgG of $1 / 5120$ confirming N the diagnosis. CT scan showed a hypodense lesion of the entire right temporal lobe extending to the temporal basal region causing deviation of mid line structures and $\omega$ severe compression of the right lateral ventricle: there? 
were also signs of left brain stem haemorrhage and compression of basal cisternac. He continued to be deeply comatose, but no longer required ventilatory support. In view of the clinical state and radiological features, the prognosis was considered such that, in consultation with his family, it was decided to stop specific therapy.

On 9 August he was breathing spontaneously, but comatose with no apparent brain stem reflexes. Tracheostomy was performed on 12 August. His tracheostomy site became infected yielding growths of Staphylococcus aureus and Pscudomonas, and the tracheostomy tube was changed several times.

He was casevaced to the United Kingdom on 26 September, still comatose but breathing spontaneously with a tracheostomy in situ, the tube again needing to be changed several times over the subsequent weeks.

A further CT scan performed in October showed widespread cortical atrophy, dilatation of the whole ventricular system and a communicating hydrocephalus.

Lumber puncture showed a pressure of $12 \mathrm{~cm} \mathrm{H} 2 \mathrm{O}$. It was felt that insertion of a shunt to treat the hydrocephalus would not be of value.

$\mathrm{He}$ developed increasing respiratory distress with progressive bronchospasm and central airways obstruction unresponsive to therapy. He died on 14 November about $3 \frac{1}{2}$ months after his initial illness.

\section{Autopsy findings}

\section{Case l}

External examination revealed a thin young adult white male. Bronchopneumonia was present in both lungs which was the final cause of death.

The brain and spinal cord were examined after fixation. The brain weighed $1310 \mathrm{~g}$.

Externally the striking feature was marked atrophy and yellow pigmentation of both temporal lobes, more severe on the left. The olfractory bulbs and mammillary bodies were softened and small. On the left side there was softening around the dorsal end of the Sylvian fissure involving the parietal-temporal region over an area of $2.5 \mathrm{~cm}$.

Coronal slices revealed marked atrophy and softening of both temporal lobes most marked in the medial regions and including the superior and middle temporal gyri on the left, involving both cortex and underlying white matter. The insular region and cingulate gyrus on either side, and on the left the adjacent inferior surface of the inferior frontal gyrus were also soft. The ventricles were dilated.

Cut surface of the spinal cord showed softening of both grey and white matter.

Histology showed the cerebral tissue to be replaced in the necrotised areas by masses of macrophages and proliferating vessels cuffed by lymphocytes. Adjacent areas showed a reactive astrocytosis, haemosiderinladen macrophages and a fine granular calcification.
Viral inclusions were not seen. In the spinal cord, degenerative changes were seen in crossed and uncrossed pyramidal tracts and in the anterior spino-thalamic and spino-cerebellar tracts, some of the changes being consistent with Wallerian degeneration.

Overall the findings were of a severe necrotising encephalitis affecting the limbic system bilaterally.

\section{Case 2}

External examination revealed a thin young adult white male with a tracheostomy in situ. There was a near-occlusive stenosis of the larynx at about the level of the tip of the trachestomy tube (Fig 1). Thick mucoid of secretions were present distal to this and also plugging the bronchial tree throughout the lungs. Cut surface of the lungs was congested but not obviously infected. A swab taken from the plugs yielded a growth of Klebsiella species. The final cause of death was regarded as respiratory failure consequent upon the tracheal stenosis and explaining the severe bronchospasm and central obstruction prior to death.

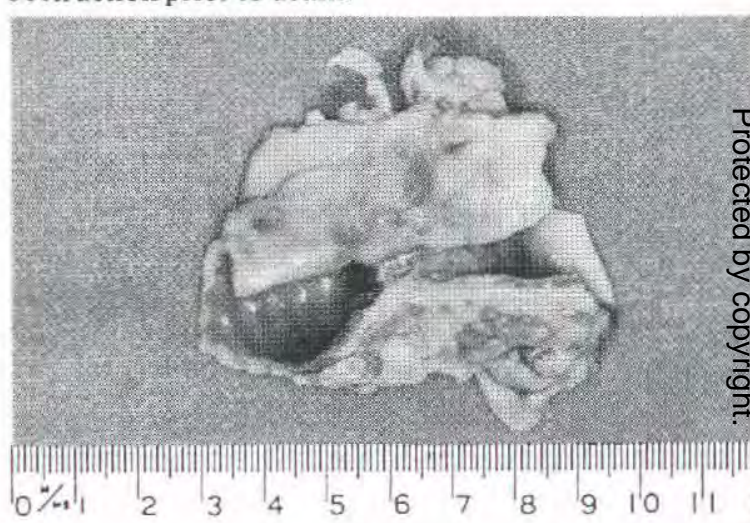

Fig 1. Almost occulusive tracheal stenosis just distal to tip of tracheostomy tube (removed).

The brain and spinal cord were examined after fixation. The brain weighed $1388 \mathrm{~g}$.

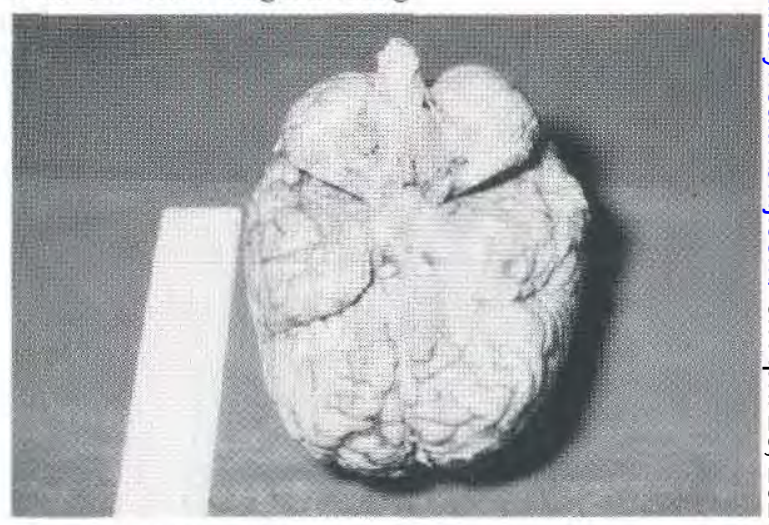

Fig 2. Undersurface of brain showing markedly shrunken right temporal lobe. 


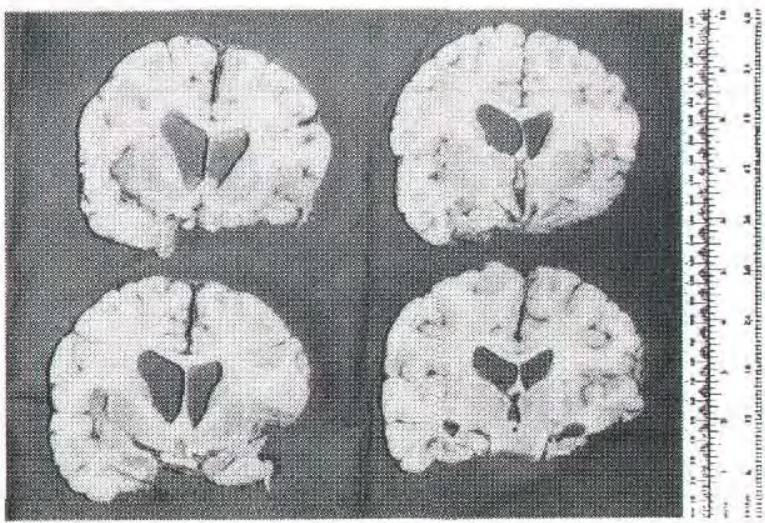

Fig 3. Coronal slices through brain showing extensive necrosis, loss of substance of temporal lobes, right more than left.

Externally, the leptomeninges appeared opaque and thick, but the striking feature was softening of both temporal lobes, especially the right (Fig 2). Coronal slices revealed marked necrosis of the temporal lobes, especially right, with loss of brain substance (Fig 3 ). The necrosis on the left was mainly confined to the hippocampus, para-hippocampal gyrus and inferior temporal gyrus. The ventral surface of the right inferior frontal gyrus was also softened. Patchy necrosis was seen in both cingulate gyri and the entire insular cortex on the right was necrotic. The ventricular system was dilated. There were midline linear softening in the basis pontis and degenerative changes in the long tracts of the spinal cord.

Histology revealed extensive gliosis, macrophage infiltration and lymphocytic perivascular cuffing in the necrotised areas typical of herpes encephalitis. The leptomeninges were infiltrated by lymphocytes. No definite viral intranuclear inclusions were seen.

Degenerative changes were present in the pons, medulla and long tracts of the spinal cord.

Overall the features were those of an extensive necrotising encephalitis involving almost the entire limbic system.

\section{Discussion}

Neither of these two cases were unusual in that they presented initially with prodromal symptoms suggestive of other relatively minor infective processes. In one series (5) this occurred in $48 \%$ of cases, the most important predictor of outcome being the level of consciousness at the start of therapy. Other authors $(1,3,4)$ stress the same point with improved mortality and morbidity on patients who were lethargic as opposed to comatose on commencement of treatment.

It is apparent therefore that early diagnosis is of paramount importance. Electro encephalographic (EEG) abnormalities have been found in all cases $(5,6)$ and are regarded as useful, but not diagnostic. Serology and/or brain biopsy are the two specific procedures employed at present, and in the present report Case 1 was diagnosed on biopsy and Case 2 on serology.

Whitley (4) in a review of the National Institute of Allergy and Infectious Diseases Collaborative Antiviral Study Group advocates brain biopsy in all cases immediately prior to therapy maintaining it is the most reliable diagnostic procedure. In a further review (7), this is reiterated but the value of biopsy as a diagnostic procedure in excluding herpes encephalitis from other conditions which may mimic it and which may themselves require different treatment is discussed. In that study, 432 patients underwent brain biopsy for a presumed clinical diagnosis of herpes encephalitis immediately prior to starting therapy: $195(45 \%)$ were found to be positive; of those negative $95(22 \%)$ were diagnosed on the basis of the biopsy as having another different disease process and $142(33 \%)$ remained without a diagnosis.

Of the 95 patients in which an alternative diagnosis was found, 38 were treatable. Most of these were other infections. In all 40 other viral nontreatable meningoencephalitides were identified, though many were shortlived self-limiting diseases. Togovirus infections were the most common with 16 cases. There were 8 examples of Epstein-Barr virus and one of cytomegalovirus. Amongst the non-treatable, non-infective processes, vascular diseases were the most common (11 cases), along with cases of tumour and toxic encephalopathy. In the patients who were negative for herpes encephalitis, the acyclovir was discontinued with no deleterious effects.

In a Swedish series (3) of 127 cases with a clinicallyco suspected diagnosis of herpes simplex encephalitis, a? total of 74 were excluded on the basis of either brain biopsy and/or serology and in 22 an alternative diagnosis was subsequently found. These included 10 other viral infections, three bacterial infections, 6 cases of toxic alcoholic encephalopathy and 3 cases of vascular disease. Modlin (8) reported 4 cases in children with enterovirus encephalitis (Coxsackie A or B), who on presentation were suspected of herpes encephalitis and who recovered completely within a few days.

There is no doubt that early treatment yields the best results, and the question arises whether one should wait for confirmatory investigations before starting acyclovir, a non-toxic drug with very few side effects.

Brain biopsy can yield false negatives (3). CSF serology may not reach its peak until as late as two months after onset of illness and may not be positive for up to 10-12 days after the appearance of neurological symptoms (3).

It is recommended by some authors that acyclovir therapy be commenced with a presumptive clinical diagnosis of herpes encephalitis, before it can be confirmed with laboratory investigations $(5,9)$. The therapy can be discontinued if an alternative diagnosis is found.

In the two cases described, there were delays of several days before the institution of therapy. Both were com- 
atose at the start of therapy. Both resulted in fatal outcomes: Case 1 did in fact recover from the comatose state only to be severely mentally handicapped.

Herpes simplex encephalitis is by no means common, but this report documents two cases in BAOR in recent years. It is to be expected that further cases may occur, even with the planned reduced manpower in the Forces. It is suggested that a high level of suspicion be maintained when confronted with unexpected neurological symptoms and behavioural changes in a febrile patient, especially as investigations such as EEG, herpes serology and brain biopsy may not always be immediately available in a military setting, and consideration be given to the administration of acyclovir for a presumed clinical diagnosis of herpes encephalitis.

Naturally this has to be put into perspective and its indiscriminate use is not recommended to all febrile patients, the vast majority of whom will have minor selflimiting diseases.

\section{Acknowledgement}

The author would like to thank Lt Col R Whiteoak, Lt Col N Hoad and Lt Col M World for their assistance and also Dr B Doshi FRCPath, Consultant Neuropathologist at the Brook General Hospital of her assistance in the neuropathological examination of the fixed brains, also Consultant Physicians Col J Dickinson and Lt Col N Ineson.

\section{REFERENCES}

1. PRICE R W. Neurobiology of human herpes virus infections. Critical reviews in Clinical Neurobiology 1986; 2: 61-123.

2. Whitley R J. Herpes simplex virus infections of the central nervous system. Drugs 1991; 42: 406-427.

3. Skoldenberg B, Forsgren M, Alestig K, et al. Acyclovir versus vidarabine in herpes simplex encephalitis. Lancet 1984; 2: 707-711.

4. Whitley R J, Alford C A, Hirsch M S, et al. Vidarabine versus acyclovir therapy in herpes simplex encephalitis. $N$ Engl J Med 1986; 314: 144149.

5. KenNedy P G E. A retrospective analysis of 46 cases of herpes simplex encephalitis seen in Glasgow between 1962 and 1985. QJ Med 1988; 255: 533-540.

6. Gulliford M C, Chandrasekera C P, CoOper R $\mathrm{A}$, et al. Acyclovir treatment of herpes simplex encephalitis: experience in a district hospital. Postgrad Med J 1987; 63: 1037-1041.

7. Whitley R J, Cobbs C G, Alford C A, et al. Diseases that mimic herpes simplex encephalitis. JAMA 1989; 262: 234-239.

8. Modlin J F, Dagan R, Berlin L E, et al. Focal encephalitis with enterovirus infections. Pediatrics 1991; 88: 841-845.

9. NICHOLSON K G. Antiviral therapy. Lancet $1984 ; 2 ;$ 을 736-738. 